\title{
COVID-19 Population Survey of Iran (COPSIR) study protocol: Repeated survey on knowledge, risk perception, preventive behaviors, psychological problems, essential needs, and public trust during COVID- 19 epidemic
}

\author{
Leila Janani ${ }^{1,2}$, Ahmad Hajebi ${ }^{3,4}$, Hajar Nazari $^{5}$, Neda Esmailzadehha ${ }^{5}$, Leila Molaeipour ${ }^{5}$, Fatemeh Varse $^{5}$, \\ Mehrdad Eftekhar ${ }^{4}$, Cornelia Betsch ${ }^{6}$, Seyed Abbas Motevalian*3,5 (D) \\ Received: 27 Apr 2020 \\ Published: 25 May 2020
}

\begin{abstract}
Background: The worldwide emergence and rapid expansion of COVID-19 emphasizes the need to assess the knowledge gap and to predict the disease-related behaviors and reactions during this epidemic.

Methods and design: COVID19 Population Survey of Iran (COPSIR) is a repeated cross sectional survey that will be conducted in 8 waves. In each wave, 515 Iranian adults aged 18 years or older will be randomly selected and interviewed by phone. The study waves will be performed at approximately weekly intervals. The survey tool is adapted from COSMO (COVID-19 Snapshot MOnitoring) study. This study will provide information on trends of knowledge, risk perception, preventive behaviors, psychological problems, essential needs, and public trust among Iranian adults during COVID-19 epidemic.

Discussion: The key findings of each wave will be immediately reported to the National Headquarters for Coronavirus Control to set better policies for disease control and prevention. Moreover, if a message is extracted from the results of this study that needs to be communicated to the public, it will be done through the mass media.
\end{abstract}

Keywords: COVID-19, Knowledge, Risk perception, Preventive behaviors, Psychological problems, Public trust

Conflicts of Interest: None declared

Funding: This study was funded by Iran University of Medical Sciences (grant number: 17604).

\section{*This work has been published under CC BY-NC-SA 1.0 license. \\ Copyright $₫$ Iran University of Medical Sciences}

Cite this article as: Janani L, Hajebi A, Nazari H, Esmailzadehha N, Molaeipour L, Varse F, Eftekhar M, Betsch C, Motevalian SA. COVID-19 Population Survey of Iran (COPSIR) study protocol: Repeated survey on knowledge, risk perception, preventive behaviors, psychological problems, essential needs, and public trust during COVID-19 epidemic. Med J Islam Repub Iran. 2020 (25 May);34:52. https://doi.org/10.47176/mjiri.34.52

\section{Introduction}

In December 2019, the new Coronavirus was detected in patients with viral pneumonia in Wuhan, China, by the China Centers for Disease Control and Prevention (1), and it was named as COVID-19 by the World Health Organization (WHO) on February 2020 (2). However, the rapid and sudden increase in the number of cases in Wuhan and some

Corresponding author: Dr Seyed Abbas Motevalian, motevalian.a@iums.ac.ir

1. Preventive Medicine and Public Health Research Center, Psychosocial Health Research Institute (PHRI), Iran University of Medical Sciences, Tehran, Iran

2. Department of Biostatistics, School of Public Health, Iran University of Medical Sciences, Tehran, Iran

3. Research Center for Addiction and Risky Behaviors (ReCARB), Psychosocial Health Research Institute (PHRI), Iran University of Medical Sciences, Tehran, Iran

4. Department of Psychiatry, School of Medicine, Iran University of Medical Sciences, Tehran, Iran

5. Department of Epidemiology, School of Public Health, Iran University of Medical Sciences, Tehran, Iran

6. Center for Empirical Research in Economics and Behavioral Sciences (CEREB), Media and Communication Science, University of Erfurt, Erfurt, Germany other provinces in China and outside China prompted the WHO to declare COVID-19 as a pandemic on March 11, 2020 (3). The first confirmed cases of COVID-19 in Iran were officially reported on February 19, 2020 (4). The identified cases rapidly increased, so that on March 19, 2020 the number of laboratory-confirmed cases reached 18407 and

$\uparrow$ What is "already known” in this topic:

Understanding population behaviors is the key element to stop the spread of COVID-19 during the pandemic.

$\rightarrow$ What this article adds:

COVID-19 Population Survey of Iran (COPSIR) is a repeated national cross sectional survey that will be conducted in 8 waves. This study will provide information on trends of knowledge, risk perception, preventive behaviors, psychological problems, essential needs, and public trust among Iranian adults during COVID-19 epidemic. 
Table 1. COPSIR sample size for different provinces of I.R. of Iran in each week

\begin{tabular}{lcccc}
\hline Province & $\mathrm{N}$ & Province & $\mathrm{n}$ & Province \\
\hline Alborz & 17 & Ilam & 4 & Lorestan \\
Ardabil & 8 & Isfahan & 33 & Markazi \\
Azerbaijan, East & 25 & Kerman & 20 & Mazandaran \\
Azerbaijan, West & 21 & Kermanshah & 13 & Qazvin \\
Bushehr & 8 & Khorasan, North & 6 & Qom \\
Chahar Mahaal and Bakhtiari & 6 & Khorasan, Razavi & 41 & Semnan \\
Fars & 31 & Khorasan, South & 5 & Sistan and Baluchestan \\
Gilan & 16 & Khuzestan & 30 & Tehran \\
Golestan & 12 & Kohgiluyeh and Boyer-Ahmad & 5 & Yazd \\
Hamadan & 11 & Kurdistan & 11 & Zanjan \\
Hormozgān & 12 & & & 83 \\
\hline
\end{tabular}

1284 died of COVID-19 (5). Only behavioral change can help stop the spread of this disease, and understanding behaviors is the key to change them. Several studies have been conducted in different countries; for instance, Eurobarometer on Influenza H1N1 in EU region (6) and COVID19 Snapshot Monitoring in Germany and other countries (e.g., Argentina, Denmark) $(7,8)$.

The aims of this study are to monitor knowledge, risk perception, preventive behaviors, psychological reactions, essential needs, and public trust among Iranian adult population during COVID-19 epidemic.

\section{Methods}

\section{Study design}

The COVID-19 Population Survey of Iran (COPSIR) is designed as a repeated cross sectional survey and will be conducted in 8 consecutive waves in the first week of April 2020. The study waves will be performed at approximately weekly intervals, which may be shorter or longer according to the spread of changes during this epidemic.

\section{Study population and sampling}

The study population includes all Iranian adults aged 18 years or older who have been registered in the National Integrated Health System (SIB), which includes the data of $95 \%$ of Iran's population. For each wave of the repeated survey, 515 Iranian adults will be randomly selected from SIB database proportionally to the size of each province (Table 1). Thus, the total sample size, which is assigned based on COSMO study protocol, will be 4120 participants.

\section{Survey instrument}

The study questionnaire is adapted from COSMO (COVID-19 Snapshot MOnitoring) study protocol $(1,2)$. COSMO study questionnaire was translated into Persian language. Then, 6 experts assessed the content validity of the translated version and modified it for COPSIR study questionnaire. Essential needs related to COVD-19 epidemic were added as a new dimension to the study questionnaire. The dimensions of COPSIR study questionnaire and the number of its items are summarized in Table 2.

\section{Telephone interviews}

In each province, at least 1 mental health officer will be trained to conduct the telephone interviews. In each week, interviewers in each province will be provided with a list of an average number of 16 to 17 randomly selected adults who live in the same or near provinces. If anyone refuses to participate or is unavailable in at least 3 phone calls, that person will be replaced by another adult from the same district. Based on the findings of the pilot study, each telephone interview takes approximately 25 to 30 minutes. The interviews will be conducted in 2 consecutive days (Sunday and Monday) in each week. The nationwide 4030 Call Center, which has recently been developed to improve public awareness and provide telephone consultations about COVID-19, will be used to facilitate the telephone interviews.

\section{Quality control procedures}

After completion of each of the 8 waves of telephone interviews, the collected data will be reviewed and the contradictory data will be identified and corrected. In addition, after each wave of interviews, national supervisors will contact at least $5 \%$ of the participants and ask a number of key questions again to compare with the reported responses.

\section{Ethical considerations}

The study protocol was approved by the ethics committee of Iran University of Medical Sciences (IR.IUMS. REC.1399.004). After providing a brief explanation about

Table 2. Dimensions and items of COPSIR study questionnaire (adapted from COSMO study)

\begin{tabular}{lcc}
\hline Dimension & Number of items & Item format \\
\hline Knowledge about COVID-19 & 45 & Yes/No, MCQ \\
Risk perception & 6 & 7-point Likert scale \\
Preventive behaviors & 43 & 33 items Yes/No \\
& & 10 items 7-point Likert scale \\
Psychological problems & 17 & 7-point Likert scale \\
Media trust and usage & 22 & 7-point Likert scale \\
Trust to responsible organizations & 9 & 7-point Likert scale \\
Agreement on public policies & 10 & 7-point Likert scale \\
Panic behavior/ panic buying & 12 & Yes/No \\
Rumors & 1 & String \\
Essential needs & 7 & 7-point Likert scale \\
\hline
\end{tabular}


the study objectives, interviewers will obtain verbal informed consents from all participants before the interview. All questionnaires and databases used in this study will be kept confidential.

\section{Project implementation and management}

COPSIR project was designed and will be implemented by Psychosocial Health Research Institute of Iran University of Medical Sciences, with the support and collaboration of Deputy for Health of the Ministry of Health and Medical Education and National Headquarters for Coronavirus Control.

\section{Discussion}

The findings of COPSIR project will be used for disease modelling and prediction of trend of COVID-19 in the country. After each wave of the study, a summary report and recommendations based on study findings will be presented to National Headquarters for Coronavirus. If any important message that requires public awareness is extracted from the study findings, it will be publicly announced through the mass media. Moreover, the findings will be compared with those of other countries that have used the same protocol. Finally, time series and other appropriate analysis methods will be used to analyze the trend after the 8 -wave period.

\section{Acknowledgement}

This study was funded by Iran University of Medical Sciences (grant number: 17604).

\section{Conflict of Interests}

The authors declare that they have no competing interests.

\section{References}

1. Huang C, Wang Y, Li X, Ren L, Zhao J, Hu Y, et al. Clinical features of patients infected with 2019 novel coronavirus in Wuhan, China. Lancet. 2020;395(10223):497-506.

2. World Health Organization. WHO Director-General's opening remarks at the media briefing on 2019-nCoV on 11 February 2020 . (www.who.int)

3. World Health Organization. WHO Director-General's opening remarks at the media briefing on COVID-19 - 11 March 2020. (www.who.int)

4. World Health Organization. Coronavirus disease 2019 (COVID-19): situation report, 31.20 February 2020.

5. World Health Organization. Coronavirus disease 2019 (COVID-19): situation report, 59. 20 March 2020.

6. The Gallup Organization. Flash Eurobarometer 287. Eurobarometer on Influenza H1N1. 2010. Available: http://ec.europa.eu/public_opinion/flash/fl_287_en.pdf.

7. Betsch C, Wieler L, Bosnjak M, Ramharter M, Stollorz V, Omer S, et al. COVID-19 Snapshot MOnitoring (COSMO): monitoring knowledge, risk perceptions, preventive behaviours, and public trust in the current coronavirus outbreak. Psych Archives. 2020; (published online March 3.)

8. Betsch C, Wieler LH, Habersaat K; COSMO group. Monitoring behavioural insights related to COVID-19. Lancet. 2020;S01406736(20)30729-7. 